Madeline Bach, OMS IV, Sonia Shenoi*, OMS I, Kathleen Winger, OMS III and Tami Hendriksz, DO

\title{
Healthier together: a pilot study on the implementation of a novel family centered pediatric obesity prevention program
}

https://doi.org/10.1515/jom-2020-0143

Received June 3, 2020; accepted February 7, 2021;

published online March 23, 2021

\section{Abstract}

Context: With pediatric obesity rates reaching epidemic scales across the United States, innovative research to identify key factors for successful implementation of obesity intervention programs is increasingly paramount. Project Healthy Attitudes Produces Positive Youth (HAPPY) is a family centered pediatric obesity prevention program targeting elementary age children and their families.

Objectives: To determine whether Project HAPPY interventions emphasizing social networks were successful.

Methods: This was a small scale evaluation of the Project HAPPY pilot program, conducted after school at a public elementary campus in Solano County, California. From April 2018 to May 2018, first year medical students from Touro University California College of Osteopathic Medicine served as "family navigators," guiding participants through a 5 week curriculum of didactic lessons, cooking demonstrations, and physical exercises. Eligibility requirements for Project HAPPY included basic English proficiency, confirmed enrollment of at least one child at the elementary school, and willingness to attend all study sessions. The primary outcome evaluated was a shift in attitude toward individual health. Changes in body mass index (BMI) and health behaviors were assessed as

Madeline Bach and Sonia Shenoi owned equal authorship.

*Corresponding author: Sonia Shenoi, OMS I, College of Osteopathic Medicine, Touro University California, 1234 Rosalind Road, San Marino, CA 91108-1130, USA,

E-mail: soniamarieshenoi@gmail.com

Madeline Bach, OMS IV, Kathleen Winger, OMS III and Tami Hendriksz, DO, College of Osteopathic Medicine, Touro University California, Vallejo, CA, USA secondary outcomes. Outcomes were assessed through surveys and measurements of BMI completed during Session 1 , Session 10, and 4, 6, and 8 month follow up sessions.

Results: Initial study participants included seven families, consisting of elementary school students with their siblings and parents $(n=27)$. Only four families $(n=13)$ completed the entire 5 week intervention. Over the course of the study, survey results of participants' attitudes regarding health suggested a positive trend toward self efficacy, while BMI appeared stable or increased. Participants demonstrated retention of successful health behaviors up to 8 months after the end of the intervention through qualitative reports of being "more conscious of meals and what (they) are eating," "valu[ing their] nutrition on a daily basis," "play[ing] a lot outside and inside on the weekends," "carry[ing] a water bottle," and “cook(ing) a lot." A higher percentage of respondents in the final session compared selected the option "strongly agree" to each of the following statements: "I can be healthier if my family works together" (87.5\% final session vs. $84.6 \%$ first session), "Drinking water makes me healthy” (87.5\% final session vs. $84.6 \%$ first session), "Moving my body makes me healthy" (87.5\% final session vs. $76.9 \%$ first session), "I reach my goals even when things get in my way” (62.5\% final session vs. $46.2 \%$ first session), "Eating fruits and veggies make me healthy" (100\% final session vs. $92.3 \%$ first session), "I can be healthy and enjoy my favorite foods" (87.5\% final session vs. $66.7 \%$ first session), and "I can create a healthy balanced meal” (87.0\% final session vs. $75.0 \%$ first session). Concrete data analysis was severely complicated by loss of study participants to follow up and incomplete data collection.

Conclusions: Project HAPPY showed promising indications that should be further evaluated in studies of larger scale and longer duration.

Keywords: early intervention; family health; obesity; pediatrics; prevention; primary care. 
Pediatric obesity and physical inactivity have reached epidemic proportions. Nationally, nearly one in five children today meets obesity criteria [1]. Long term health consequences associated with childhood obesity are extensive. These include diabetes, high cholesterol, hypertension, and heart disease [2, 3]. Obese children often feel isolated, predisposing them to depression, suicidal ideation, and uncontrolled eating behaviors [4, 5]. Unhealthy children often grow to be unhealthy adults, thus placing a burden on the healthcare system [6]. Some areas in the United States have disproportionally high childhood obesity rates. Solano County is one such region, with a higher rate of overweight, obese, and inactive children than the average rate in California [7].

Current research suggests that pediatric obesity prevention programs can be successful when targeted at younger children ( $<12$ years old) and continued for at least 12 weeks [8]. Meeting with groups of children at the same time can help expand the children's social networks, decrease obesity stigmas, and vitalize obesity prevention $[5,9]$. Studies [8-11] have proposed that involving families can be key in creating a sustainable environment that promotes healthy lifestyles even after completion of the program. Familial participation is a progressive idea that allows for healthy habits to be well incorporated in daily life and to persist long term [12, 13]. Research has also suggested that obesity prevention programs are more effective when delivered by dedicated interventionalists vs. classroom teachers [14]. One study [15] showed that under the guidance of interventionalists who had taken a 4 week nutrition course, pediatric participants had a significantly lower increase in BMI and obesity rate. Dedicated interventionalists have the time and energy to apply specialized training in nutrition toward not only individualized interventions for specific participant groups but also refined presentation strategies, whereas classroom teachers prioritize lesson plans and lectures, with the majority of their time taken up by attending to essential classroom logistics [16].

Based on this pool of evidence, we designed Project Healthy Attitudes Produce Positive Youth (HAPPY) as a pediatric obesity intervention program that utilized a family based approach and incorporated the unique factor of a trained medical student "family navigator." Family navigators were medical students who received interventionalist training for 12 weeks through biweekly lunchtime sessions on nutrition, exercise, diet, socioeconomic barriers to healthy lifestyle changes, and social determinants of pediatric obesity. We hypothesized that the use of family navigators would contribute to the cohesiveness of the program by providing individualized support and education for families. Each family navigator was assigned to a family for the duration of the study to create a bond and build trust. The family navigator's role was to foster a collaborative environment for dialogue, educate the family on healthy habits, and encourage the family toward healthier choices.

We sought to discover whether this family centered program, with the addition of family navigators who had interdisciplinary and culturally competent nutrition training, would lead to success with instilling healthy habits long term. Here, we present results from Project HAPPY's 5 week pilot program, conducted between April 2018 and May 2018, with follow up data collected through January 2019. While strong conclusions cannot be drawn from this pilot study due to Project HAPPY's small sample size and short duration, prior research studies have shown that having smaller sample sizes and a shorter duration of weeks can produce larger effects than studies that are longer in duration [15]. This is because longer interventions may be unappealing to participants, which causes them to drop out of the intervention or disengage from the program [16]. Thus, studies of smaller scale may still provide valuable data, while also offering a launch point for future studies of larger scale. With the understanding that this is a novel program, the goal of this initial Project HAPPY pilot study was to evaluate outcomes and provide insight into possible future directions for project implementation.

\section{Methods}

\section{Recruitment}

Prior to participant recruitment, this study was approved by the Institutional Review Board at Touro University-California (\#M-0178). Project HAPPY was conducted at an elementary school (kindergarten through sixth grade) in Solano County, California. Study participants included students and their families. Eligibility requirements included basic English proficiency, one member of the family enrolled as a student at that elementary school, and willingness to attend all didactic and follow up sessions.

The goal was to recruit 40 study participants or 10 families. Twenty families were assessed for interest in the program at the school's Family Night, held in March 2018. School administrative staff assisted with program outreach to recruit families. Family navigators contacted potential families by email or phone to disclose the study goals and timeline.

Family navigators reviewed consent and assent documents prior to the first session in person with potential participants. Participants were informed of the total commitment of 21 "in person" hours, including 10 didactic sessions and three follow up sessions, with the opportunity to make up a maximum of two missed sessions. 


\section{Compensation}

There was no cost to participants to partake in Project HAPPY. Families were given two $\$ 50$ gift cards as incentive to attend sessions and buy groceries to make the recipes presented in the cooking demonstrations. Families received a $\$ 25$ gift card after each follow up session. Total monetary compensation was $\$ 175$ per family. Additionally, participants were provided with water bottles, tote bags, herb gardens, activity pages, sticker charts, and other materials necessary for lessons.

Families must have attended or completed make up sessions with their family navigators for gift card compensation, which was given at the end of the program. If a family chose to discontinue involvement in the study, compensation was provided only for the portion of the program completed.

\section{Program description}

The objective of Project HAPPY was to provide a community based, family centered educational series that taught positive psychosocial development, nutrition, and exercise habits to children and their families. The curriculum was designed by Touro University California College of Osteopathic Medicine medical students under the direction of a board certified pediatrician (T.H.).

As 12 weeks had been touted as a successful program length in a prior study [16], the pilot study was originally designed as a 12 week program with participants meeting weekly as a group. Due to the school's scheduling availability, the pilot program was compressed to 10 sessions over 5 weeks. These sessions occurred every Tuesday and Thursday from April 17th, 2018 to May 18th, 2018. Each $1.5 \mathrm{~h}$ session was broken down into 20 min of physical activity, 40 min of didactic lessons, and $30 \mathrm{~min}$ for families to check in with their respective family navigators. On Thursdays, the didactic session included a 20 min cooking demonstration, with less time spent on the family navigator check in. The topics of the 10 sessions were: introduction/herb gardens/family commitments; goals and barriers; moving minutes; water; fruits and vegetables; mindful eating; treat yourself; meal planning; fiber; and review/wrap up.

The 5 week intensive program was planned to be followed by four follow up sessions, spaced quarterly over the following year. Due to scheduling conflicts, the number of follow up sessions was reduced to three sessions at 4 months, 6 months, and 8 months after the end of the program.

\section{Study measurements}

The primary outcome evaluated in this study was a change in attitudes regarding activity level, nutrition, and water consumption. Baseline measures included responding to 10 statements on a 5 point Likert scale, with children given an associated cartoon character displaying emotions for easier interpretation.

Two secondary outcomes were assessed: behavioral changes and change in body mass index (BMI) for adults or BMI percentile for children. All participants, both adults and children, were asked to complete the surveys and measurements. Measurement of behavioral changes included a nutrition recall in which participants were asked to recall what food they ate in the past $24 \mathrm{~h}$ and an exercise recall in which participants were asked to recall what exercise they completed in the past $24 \mathrm{~h}$. Measurement of body composition changes included measuring weight by scale and height by tape measure, done individually and privately by family navigators in a separate room. BMI was calculated for adults as weight in kilograms divided by height ${ }^{2}$ in meters [2]. BMI percentile was determined for children using sex specific BMI-for-age CDC growth charts to account for body composition variability between sex and age [17].

Collection of data on primary and secondary outcomes was completed during session 1, session 10, and at all follow-up sessions. All participant information and data were kept in a locked cabinet to ensure compliance with Health Insurance Portability and Accountability Act privacy regulations.

\section{Results}

Seven families, including a total of 27 individuals, provided informed consent and attended the first session on April 17, 2018. By the last session, 51.8\% of participants (14 participants from three families) were lost to follow-up due to cited schedule conflicts. Thirteen participants from four families completed the entire 10 session intervention and were included in data analysis. Only six participants from two families (22.2\%) attended the final follow up session at 8 months. Table 1 provides demographic data for the 13 participants who completed the program. Ethnicity was self reported.

\section{Primary outcome}

Evaluation of attitude changes toward individual health was completed through 10 statements rated by participants on a 5 point Likert scale. The 10 statements polled were positive self affirmations regarding nutrition, exercise, and barriers to health. Results were calculated by percent of responses at each Likert scale score for each statement during Session 1

Table 1: Demographic data of Project HAPPY participants.

\begin{tabular}{llll}
\hline Child participants & \multicolumn{2}{l}{ Adult participants } \\
\hline Age, years & $\mathbf{n}$ & Age, years & $\mathbf{n}$ \\
$<5$ & 3 & $<30$ & 3 \\
$5-12$ & 6 & $>30$ & 1 \\
Ethnicity & & Ethnicity & \\
Black & 2 & Black & 1 \\
Latino & 3 & Asian & 1 \\
Multiracial & 4 & Hispanic & 2 \\
Body Mass Index & & Body Mass Index & \\
Underweight (<5th percentile) & 1 & Underweight (<18.5) & 0 \\
Normal (5th-85th percentile) & 5 & Normal (18.5-24.9) & 1 \\
Overweight (85th-95th percentile) & 1 & Overweight (25.0-29.9) & 1 \\
Obese (>95th percentile) & 2 & Obese (>30.0) & 2 \\
\hline
\end{tabular}


(initial session) and Session 10 (final session) from the four families that completed the study (Figure 1). A total of 126 responses were recorded during Session 1, but only 80 responses were recorded during Session 10, due to missed attendance by one family and incomplete responses to the 10 statements by the families that were present.

A higher percentage of respondents in the final session compared to the first session selected the option "strongly agree" to each of the following statements: "I can be healthier if my family works together" (7 of 8 [87.5\%] final session vs. 11 of 13 [84.6\%] first session), "Drinking water makes me healthy" (7 of 8 [87.5\%] final session vs. 11 of 13 [84.6\%] first session), "Moving my body makes me healthy" (7 of 8 [87.5\%] final session vs. 10 of 13 [76.9\%] first session), "I reach my goals even when things get in my way" ( 5 of 8 [62.5\%] final session vs. 6 of 13 [46.2\%] first session), "Eating fruits and veggies make me healthy" (8 of 8 [100\%] final session vs. 12 of 13 [92.3\%] first session), and "I can be healthy and enjoy my favorite foods" (7 of 8 [87.5\%] final session vs. 8 of 12 [66.7\%] first session), and "I can create a healthy balanced meal" (7 of 8 [87.5\%] final session vs. 9 of 12 [75.0\%] first session). Concrete data analysis was severely complicated by loss of study participants to follow-up and incomplete data collection.

In Session 1, each statement had at least one participant who had marked "neutral," "disagree," or "strongly disagree." There were five statements for which participants had answered "strongly disagree," with the most dissent expressed with the statement "I can reach my goals even when things get in the way." In session 10, there were no dissenting responses and only two statements with neutral responses: "I connect with others when we eat a meal together" and "I reach my goals even when things get in my way."

\section{Secondary outcomes}

BMI demonstrated an average trend, stable trend, or increasing trend for both children and adults. By the 6 and 8 month follow-up sessions, changes were apparent from baseline demographic data (Figure 1). One adult moved from the "overweight" to "obese" category; one child increased from "underweight" to "normal weight"; and two children increased from "normal weight" to "overweight." It must be noted that there were several large outlier data points, as well as missing measurements due to loss of participants to follow up. No definitive conclusions regarding the study's effect on BMI could be drawn.

To evaluate trends in BMI data for adults and BMI percentile for children, values were examined using line
A

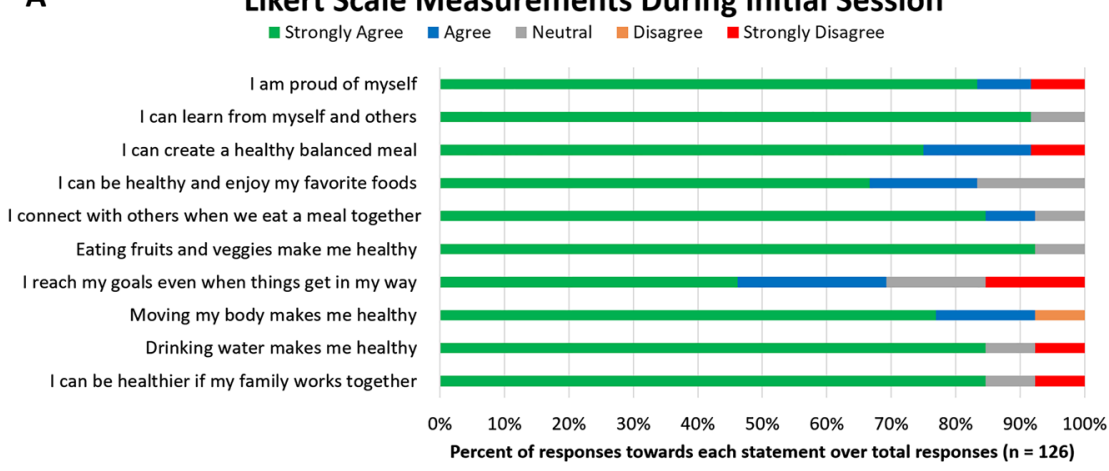

8

Likert Scale Measurements During Final Session

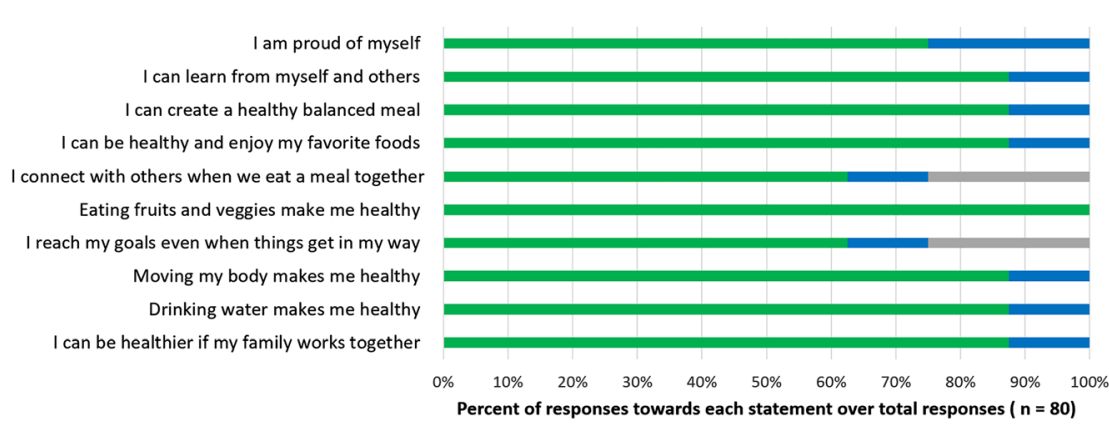

Figure 1: Surveyed participant attitudes towards health taken during $(A)$ initial session 1 and (B) final session 10, by percent of participant responses on a Likert scale. 
graphs (Figure 2). Missing data points were noted due to incomplete attendance.

To evaluate changes in health behaviors, qualitative data was recorded through surveys during follow up sessions. Participants were asked what habits they retained from Project HAPPY, and how they supported each other as a family to meet health goals. A total of 46 responses were given over the course of three follow up sessions, with a representative sample of participants' comments displayed in Table 2.

An analysis of the qualitative responses collected from program participants revealed repetition of several common themes reflecting an understanding of core session topics. These themes include increased exercise, increased water intake, and eating more healthfully. Participants demonstrated retention of successful health behaviors up to 8 months after the end of the intervention through qualitative reports of being "more conscious of meals and what [they] are eating," "valu[ing their] nutrition on a daily basis," "play[ing] a lot outside and inside on the weekends," "carry[ing] a water bottle," and "cook[ing] a lot."

\section{Discussion}

In comparing changes in health attitudes from the initial to the final session, most participants at the end of the program agreed that exercise and eating fruits and vegetables contributed to their health. However, a definitive conclusion on the primary outcome could not be reached due to incomplete session attendance.

In evaluating the secondary outcome of changes in health behavior, Project HAPPY showed great promise with positive responses collected on healthy attitudes toward core session topics (Table 2). Participants cited implementation of behavioral changes encouraged in Project HAPPY, even up to the 8 month follow up session after the end of the program. In their responses, families stated willingness to buy more organic fruits and vegetables while counting calorie intake, consistency in selecting healthy meals if eating at a restaurant, the importance of leaning on each other for support in choosing healthier options, and willingness to increase outdoor activities. These affirmations suggested that Project HAPPY made an
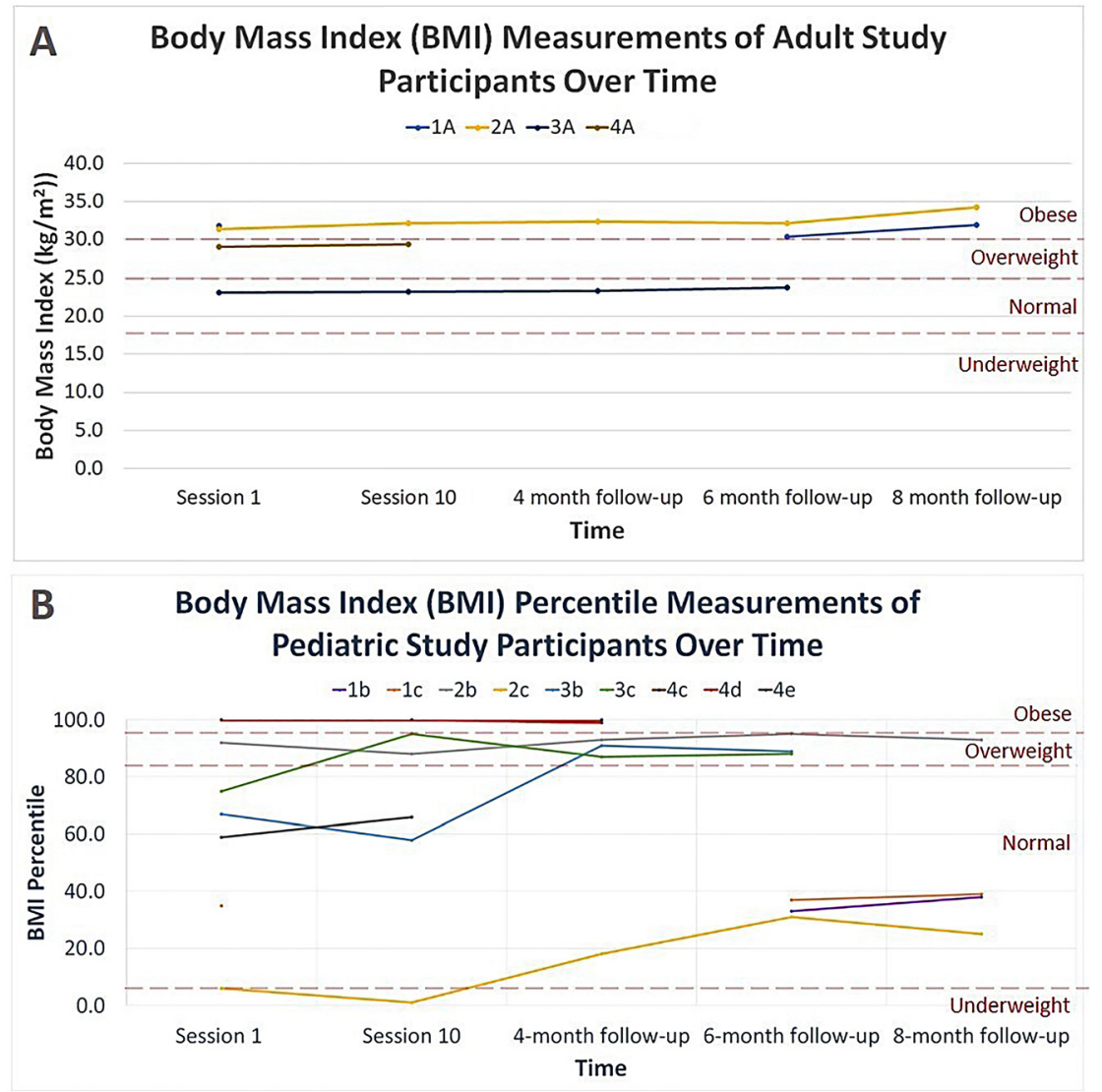

Figure 2: Calculated $(A)$ body mass index (BMI) of adult and (B) BMI percentiles of pediatric study participants over the course of the study. To deidentify data, each participant was labeled with a number representing their family and a letter pertaining to the individual, with " $A$ " designating the parent and "b" through "e" each designating a child in the family. 
Table 2: Representative sample of participant responses collected during 4 and 8 month follow up sessions.

\begin{tabular}{|c|c|c|}
\hline \multirow[t]{2}{*}{ Question } & \multicolumn{2}{|c|}{ Sample response } \\
\hline & Children & Adults \\
\hline $\begin{array}{l}\text { Can you tell me one thing you did after } \\
\text { Project HAPPY that you would not have } \\
\text { done before? }\end{array}$ & $\begin{array}{l}\text { "Play a lot outside and inside on weekends" } \\
\text { "Carry a water bottle" } \\
\text { "Trying new foods because of the "two bites } \\
\text { rule"” }\end{array}$ & $\begin{array}{l}\text { "We began buying more organic fruits and vegetables } \\
\text { while counting our calorie intake. We stayed away from } \\
\text { sugary cereals and shakes" } \\
\text { "Added new rule: cleaning up after themselves after they } \\
\text { eat" } \\
\text { "Value my nutrition intake on a daily basis" }\end{array}$ \\
\hline $\begin{array}{l}\text { Can you tell me one thing that was } \\
\text { harder than you expected? }\end{array}$ & $\begin{array}{l}\text { "Harder to play outside, not a lot of space" } \\
\text { "Trying new foods" }\end{array}$ & $\begin{array}{l}\text { "Staying consistent with purchasing healthy meals if I } \\
\text { am eating out" } \\
\text { "Exercise - timing, hard to fit into routine, weather } \\
\text { limitations" }\end{array}$ \\
\hline $\begin{array}{l}\text { How have you and your family sup- } \\
\text { ported each other? }\end{array}$ & $\begin{array}{l}\text { "By talking at the table while we eat" } \\
\text { "Helping sister drink water by calling it } \\
\text { "princess water" } \\
\text { "Encouraging brother to run outside" }\end{array}$ & $\begin{array}{l}\text { "We keep each other accountable in choosing healthier } \\
\text { options" } \\
\text { "We have mindful eating and we have dinner together } \\
\text { instead of eating it in front of the TV" } \\
\text { "Anytime we are cooking we try to help as much as we } \\
\text { can" }\end{array}$ \\
\hline $\begin{array}{l}\text { Can you tell me something you learned } \\
\text { from Project HAPPY? }\end{array}$ & $\begin{array}{l}\text { "Having more exercise, eating more fruits } \\
\text { and veggies, and drinking more water } \\
\text { makes me healthy" } \\
\text { "How to cook" } \\
\text { "Egg in bowl with syrup around it makes the } \\
\text { egg get sick. If you drink water, your urine is } \\
\text { clear" }\end{array}$ & $\begin{array}{l}\text { "Our days are more positive when we keep tight in- } \\
\text { teractions with each other" } \\
\text { "The value of what we put inside of our bodies and the } \\
\text { perks with taking care of ourselves" } \\
\text { "To choose healthier meals. We are more active. My kids } \\
\text { drink more water" }\end{array}$ \\
\hline
\end{tabular}

impact on health knowledge and attitudes for the families who participated through the 5 weeks of sessions. These results attested to the importance of approaching pediatric obesity with respect to the osteopathic tenet of viewing the person as a unit of body, mind, and spirit. By nurturing close relationships between children and their families, we hope to have helped equip families with healthy habits that may help decrease the stigma of pediatric obesity in their own lives and to have given them skills to practice healthy habits that may result in better physical and mental resilience in children.

\section{Limitations}

Project HAPPY's small sample size was both a strength and limitation. As a strength, it allowed for individual attention to be paid to participants, which was helpful for the development and growth of healthy habits. In addition, the role of family navigators in our study was significant and deserving of further investigation in future studies. By supportively engaging children in activities, family navigators proved essential in providing individualized attention to pediatric participants. Families, including adults and children, reported multiple positive aspects of having family navigators. Future studies will focus on transitioning the program into a yearly service based learning elective for Touro University California College of Osteopathic Medicine medical students. The program's effect on family navigators is an outcome that we are interested in assessing in future cohort studies. Other outcomes that could be evaluated for family navigator participants include medical knowledge, cultural competency, communication with vulnerable populations, community based research skills, and development of entrustable professional activities.

While standardized Likert scales were used to assess the primary outcome, it was difficult to ascertain whether younger participants were influenced by family members or other children near them as they completed their Likert scales. The importance of complete data collection was not emphasized to family members or family navigators, resulting in information gaps from incomplete answers. Height and weight measurements were subject to operator variability and inexplicable outlier BMI data points. Suggestions for future cohorts to increase data quality include standardized family navigator training on unbiased elicitation of information, separating participants during data collection to avoid influenced answers, collecting 
measurements performed by the same navigator to decrease operator variability, and standardizing the data record for comparison to prior measurements, including rechecking outlier points.

Another significant limitation in our study was the small sample size, along with loss of participants to followup. This limited use of statistical analysis and power for the study. To increase both participant recruitment and retention, future recruitment efforts could involve full disclosure of the program schedule and written consent to commit to the program's demands. We believe a greater focus could also be placed on building rapport between families and family navigators, which we believe might be leveraged into consistent attendance. Family navigators could lead warm up games and lessons with their respective participant families that may occur outside the group setting, such as before or after class, to strengthen relationships. Family navigators could also conduct weekly phone calls to provide presession reminders and discuss attendance management.

Our pilot study was compressed to 5 weeks due to scheduling conflicts, which may have contributed to low participant retention due to a sudden biweekly schedule. Future cohorts could be conducted in weekly sessions over 12 weeks, with supplementary check-ins by family navigators on families as needed. By extending program length, more significant differences in health attitudes and habits may also be observed.

In summary, multiple aspects of the planned study were altered, including number of sessions, program length, and follow-up data collection. Statistical analysis was not applied due to limited data. These limitations deeply affect the conclusions that can be drawn from our data. It is imperative that future executions of Project HAPPY increase data quality in number of sessions, program length, followup strategies, and adequate data for statistical analysis.

\section{Future studies}

Given the limited data and adjustment to multiple aspects of the study, further studies of larger scale and longer duration are essential to better evaluate the outcomes studied in this pilot program. In addition, future studies can evaluate Project HAPPY as a service based learning program and analyze the impact of serving as a family navigator. As of this time, Project HAPPY has received grant funding for continuation and expansion of the program, with the eventual hope of implementing Project HAPPY as a permanent health based intervention program in elementary schools within Solano County. It is our goal to perform yearly evaluations for continual feedback and improvement.

\section{Conclusions}

As a family focused pediatric obesity prevention program with family navigators, Project HAPPY showed promising indications in creating and retaining healthy attitudes and behaviors among participant families in this pilot assessment. Project HAPPY's key findings have clinical applications in that physicians should encourage families to focus on cooking, eating, and exercising together to decrease stigma and ingrain healthy practices in children, thus leading to healthier lifestyles.

Acknowledgements: The authors thank Jay Shubrook, DO, for study review; Katherine Yu, DO, for coordination of the pilot program; and Touro University California family navigators Jaimie Long, Kavitha Gilroy, Liana HindsPereira, Pranay Bonagiri, Rowenna Hann, and Souad Hamade for curriculum design, program implementation, and data collection.

Research funding: Project HAPPY was initially funded by a grant from the Sutter Health Community Benefits Program. Funds were used to purchase supplies, including t-shirts for facilitators, food for the cooking demonstrations, paper, pens, participant compensation (gift cards) and participant incentives (such herb gardens and water bottles). Sutter Community Benefits did not participate in any portion of the study design, data collection/analysis/interpretation, or manuscript preparation.

Author contributions: Ms. Bach provided substantial contributions to conception and design, acquisition of data, or analysis and interpretation of data; Ms. Shenoi drafted the article or revised it critically for important intellectual content; Ms. Winger and Dr. Hendriksz gave final approval of the version of the article to be published; and all authors agree to be accountable for all aspects of the work in ensuring that questions related to the accuracy or integrity of any part of the work are appropriately investigated and resolved.

Competing interests: None reported.

Informed consent: All participants in this study gave informed consent.

Ethical approval: This study was approved by the Institutional Review Board at Touro University-California.

Disclaimer: Dr. Hendriksz, a Journal of Osteopathic Medicine Associate Editor, was not involved the peer review of this manuscript or the decision to publish it. 


\section{References}

1. Centers for Disease Control and Prevention. Childhood obesity facts [Online]. Available from: https://www.cdc.gov/ healthyschools/obesity/facts.htm [Accessed 30 Sep 2020].

2. Narang I, Mathew JL. Childhood obesity and obstructive sleep apnea. J Nutr Metabol 2012;2012:134202.

3. Beck AR. Psychosocial aspects of obesity. NASN Sch Nurse 2016; 31:23-7.

4. Morrison KM, Shin S, Tarnopolsky M, Taylor VH. Association of depression \& health related quality of life with body composition in children and youth with obesity. J Affect Disord 2015;172:18-23.

5. Halfon N, Kandyce L, Slusser W. Associations between obesity and comorbid mental health, developmental, and physical health conditions in a nationally representative sample of US children aged 10 to 17. Acad Pediatr 2013;13:6-13.

6. Gordon-Larsen P Longitudinal trends in obesity in the United States from adolescence to the third decade of life. Obesity 2010; 18:1801-4.

7. Solano County Overweight, obesity and physical inactivity rates [Solano County website]. Available from: https://www. solanocounty.com/civicax/filebank/blobdload.aspx? BlobID=26174 [Accessed 30 Sep 2020].

8. Davis AM, Daldalian MC, Mayfield CA. Outcomes from an urban pediatric obesity program targeting minority youth: the healthy hawks program. Child Obes 2013;9:492-500.

9. Janicke DM, Steele RG, Gayes LA. Systematic review and metaanalysis of comprehensive behavioral family lifestyle interventions addressing pediatric obesity. J Pediatr Psychol 2014;39:809-25.

10. Styne DM, Arslanian SA, Connor EL. Pediatric obesityassessment, treatment, and prevention: an endocrine society clinical practice guideline. J Clin Endocrinol Metab 2017;102: 709-57.

11. Sung-Chan P, Sung YW, Zhao X, Brownson RC. Family-based models for childhood-obesity intervention: a systematic review of randomized controlled trials. Obes Rev 2013;14:265-78.

12. Pollock NK. Childhood obesity, bone development, and cardiometabolic risk factors. Mol Cell Endocrinol 2015;410: 52-63.

13. Africa JA, Newton KP, Schwimmer JB. Lifestyle interventions including nutrition, exercise, and supplements for nonalcoholic fatty liver disease in children. Dig Dis Sci 2016;61: 1375-86.

14. Baranowski T, Cullen KW, Nicklas T, Thompson D, Baranowski J. School-based obesity prevention: a blueprint for taming the epidemic. Am J Health Behav 2002;26:486-93.

15. Rosario R, Oliveira B, Araujo A. The impact of an intervention taught by trained teachers on childhood overweight. Int J Environ Res Publ Health 2012;9:1355-67.

16. Stice E, Shaw H, Marti CN. A meta-analytic review of obesity prevention programs for children and adolescents: the skinny on interventions that work. Psychol Bull 2006;132:667-91.

17. Center for Disease Control and Prevention. Clinical growth charts [Online]. Available from: https://www.cdc.gov/growthcharts/ clinical_charts.htm [Accessed 30 Sep 2020]. 